\title{
Zalabardo on Semantic Unity and Metaphysical Unity
}

\author{
Colin Johnston, University of Stirling
}

1.

In his paper 'The Tractatus on Unity', José Zalabardo argues that the Tractatus makes important contributions towards the resolution of two related problems, the problem of semantic unity, and the problem of metaphysical unity. The problem of metaphysical unity consists in explaining how the unity of a fact arises out of the multiplicity of its constituent objects. The problem of semantic unity consists in explaining how a propositional representation doesn't merely introduce various objects but furthermore represents them as combined in a certain way. According to Zalabardo, Wittgenstein takes rather different attitudes to these two problems. The semantic problem is solved by the idea, central to Wittgenstein's picture theory, that a representation is a fact which exemplifies in its own combinatorial mode the way in which it represents its objects as combined. The metaphysical problem, by contrast, is rejected as relying on a mistaken conception of facts as compounds. In this response, I take individual issue with both attributions, and briefly query also their coherence.

2.

"The semantic problem of unity," Zalabardo writes, "arises for a [certain] strategy for explaining our faculty of propositional representation" (3). According to this strategy:

language and the mind make contact with the world in the first instance at the level of the items from whose combination facts are produced. Thus, for example, a mental or linguistic item that represents Pavarotti as singing is connected in the first instance to the individual Pavarotti and the property of Singing. Then the representational item will represent the world as containing a fact produced by the combination of the individual Pavarotti and the property of Singing. ... The [semantic problem of unity] arises from the reflection that singling out the individual and the property is not enough for representing things as being a certain way-for representing Pavarotti as singing. ... The problem consists in explaining what else will be required for representing things as being a certain way in mind or language, besides singling out the items. (3)

A representation doesn't merely single out various things: it furthermore represents those things as combined in a certain way. But how? Well, Wittgenstein's basic proposal, Zalabardo maintains, is that the representation is not a mere collection of names introducing a collection of objects, but a fact which exemplifies in its own mode of combination the way in which the objects are represented as combined. "By using a fact to represent things as being a certain way," Zalabardo writes, "we bring a mode of combination into the representational episode" (7). And this mode is precisely that in which the objects are represented as combined: "we represent things in the world as combined with one another in the same way in which the constituents of the depicting fact are combined with one another." (7) 
This matter of semantic unity is sometimes cast by Zalabardo as a question not of how we represent, or of how a representation represents, but of how a representation is understood. In this guise, the problem becomes that of "explaining how, in an episode of understanding, the subject is aware [not merely of various objects but also] of the way in which [those objects] are represented by the proposition as combined with one another" (5). Here the proposed solution is that in understanding a proposition we do not merely bring to mind the items referred to by its various names, but recognise also the way those names are combined within the representation. "We bring the mode of combination to consciousness by grasping the fact that we use as a picture and its structure - the way in which its constituents are combined with one another." (9)

As Zalabardo points out, such a 'grasping the fact that we use as a picture' will need to be a species of awareness distinct from, and indeed prior to, the propositional representation/understanding it wants to explain. Zalabardo's exegetical proposal for "this more basic type of grasp" (9) is that it is "provided in Wittgenstein's framework by the notion of showing" (9). I'm not interested to criticize this specific suggestion. What I want to do, rather, is query what is underlined by the talk here of 'more basic', namely that Zalabardo sees Wittgenstein as offering what might be called a reductive account of propositional understanding, or again of propositional representation. The proposition's expression of a sense, its representing certain objects as combined in a certain way, is to be explained with appeal to two theoretically prior ideas. First there is 'singling out', or reference. But this is not enough, so appeal is made also to an idea of the exemplification of a mode of combination. Or as regards understanding, this cannot be explained by appeal only to the recognition of relations of reference, but must draw also on a grasping of the representation's combinatorial mode.

It is surprising given the drift of much recent Tractatus scholarship that Zalabardo doesn't consider the question whether Wittgenstein is interested in providing a reductive account of the operation (or understanding) of a proposition. Zalabardo discusses Wittgenstein in context with Russell, but there has been an emphasis in the literature also on Wittgenstein's inheritance from Frege. In particular, it is now I think a steady orthodoxy that Wittgenstein's adoption of Frege's context principle is such that reference is not theoretically prior in the Tractatus to the expression of sense. Reference is understood by Wittgenstein only as an aspect, or element, of sense expression. In a similar vein, Zalabardo assumes without hesitation that the notion of a fact's mode of combination, and so that of a fact, is theoretically prior for Wittgenstein to propositional representation, and so theoretically prior to truth. And whilst this assumption holds good of course for Russell, it is false for Frege and will be false on a more Fregean reading of the Tractatus (see, e.g. Sullivan 2005). Personally, I think that the Russellian realism of fact as prior to truth is never in view, even, for the Tractarian Wittgenstein. His concerns lie elsewhere. But in any case, the smaller complaint is that in taking Wittgenstein to solve rather than reject the problem of semantic unity, Zalabardo implicitly and unquestioningly rejects a prevalent interpretation under which the "the reflection that singling out ... is not enough for representing things as being a certain way" (3) is sorely misguided. Naming, or "bringing an object to consciousness', is not some already-given act which fails to be enough for propositional 
representation. Rather, naming has no status other than as an aspect of propositional representation, so that spelling out its nature will amount to nothing less than an articulation of such representation.

\section{3.}

Another channel through which skepticism can be voiced about Zalabardo's reductive interpretation would focus on his central proposal of 'in the same way'. According to Zalabardo's Wittgenstein, a proposition presents objects, and it has its mode of combination, and together these amount to its representing that the objects are so combined. But how? Why? Why should a fact whose elements stand for various objects represent that those objects are combined in the same way as its elements?

This concern could be read as a request for elaboration. How does this get to constitute that? Or it could be read as a request for justification as against potential rivals. Why should the proposal of an identity of mode be preferred to one of a correlation of modes? Why should we not hold that objects introduced by certain names at certain positions in a mode of combination constitute a representation of those objects as occupying distinct but correlated positions in a distinct but correlated mode? At base, though, the concern is not that Zalabardo's proposal on Wittgenstein's behalf is in some way insufficiently explained or justified, but a sense that as Wittgenstein would see it, this is not somewhere to be making 'proposals'. There is for Wittgenstein, one might think, no intelligible question given a proposition of how it represents its objects as combined, no intelligible question which might be answered by an 'in the same way' claim. Rather, the proposition's mode of combination is in itself a mode of a proposition which represents objects as so combined. One has the propositional mode of combination in view, and so the proposition in view, only insofar as one has in view, as such, the way such propositions represent objects as combined. Now this attribution will of course itself need elaboration and exegetical justification - something for which there is no space here. Evidently, though, it is incompatible with Zalabardo's reductive perspective, a perspective witnessed in repeated talk of "facts that play the role of propositions" $(14,17)$ - as if a proposition's status as a proposition were incidental to it, as if there were no distinction in Wittgenstein between sign and symbol.

\section{4.}

Let's move on to the problem of metaphysical unity. This is the problem of how the unity of facts "arises from the manifold of their constituents" (12). Unlike the problem of semantic unity, this is not something Wittgenstein is held to solve. Rather, he rejects a presupposition of its arising:

According to the Tractatus, facts in general, and the facts that play the role of propositions in particular, should not be construed as compounds. They don't arise from the combination of constituents. They are ultimate, indivisible units. On this account, there simply isn't a problem of how the unity of facts and propositions arises 
from the multiplicity of their constituents. Wittgenstein doesn't have a problem with metaphysical unity not because he has found a way of saving CAF [- the Compositional Account of Facts -] from the difficulty, but because he rejects CAF. (14)

Here I am, I suspect, more sympathetic to Zalabardo's basic line. At the same time, however, I'm unclear what the line is, because I'm unclear what CAF amounts to. And certain things Zalabardo wants to say in development his stance strike me as markedly unTractarian.

Rejecting CAF, Zalabardo says that facts are "basic units" "not produced by the combination of more simple items" (14). Names are not "constituent parts" of propositions but "features that propositions share with one another" (14). Ok, but in what sense of 'basic' or 'simple'? In what sense of 'production'? In what sense of 'feature'? I wasn't able to gather clear answers to these questions. The discussion earlier in the paper of semantic unity depended for its sense on an idea of a fact as a combination of objects, of objects as constituents of facts, of a fact has having a mode of combination etc. Following Wittgenstein's lead, Zalabardo's discussion was shot through with such talk, and there is no suggestion now that the earlier work is being undermined. So what is the difference between a fact having objects as constituents and a fact having objects as constituent parts? What is the difference between facts being combinations of objects and facts being produced by the combination of objects?

At times, Zalabardo seems to suggest that what he wants to oppose is the idea of a temporal process of objects combining. So he insists that "no combination needs to take place" (16):

The unity of a proposition is non-derivative, fundamental. It doesn't arise from a process of composition. (16)

But of course a Tractarian fact is not the result of a temporal process of composition, something that takes place, any more indeed than a Russellian fact is the result of such a process! Opposing that is hardly a substantive position. Presumably, then, Zalabardo's talk of 'process' and 'taking place' is a metaphor: but what for? On 'features' versus 'compounding', Zalabardo writes:

Just as people share heights, incomes, hobbies and character traits without being compounded from these items, propositions [and facts more generally] share characteristic marks without being compounded from them. (14)

But this comparison isn't helpful either. An atomic fact is set forth, and completely so, by the elementary proposition which expresses it. And within such an expression there is reference to, and only to, its constituent objects. Quite unlike the matter of people's heights and incomes, that is to say, there is no fact beyond its 'features' or 'characteristic marks', nothing to which those 'features' attach. A fact has no content, or indeed particularity, beyond that of its objects. So Zalabardo's "Just as" here must read as a "Somewhat similarly". But what is the similarity to be?

To be clear, I'm not suggesting that the sentence "A fact is not produced out of 
objects" can in no way be habilitated, that Wittgenstein's position cannot be described as one on which facts are basic. On the contrary, Wittgenstein's straightforward stance here might indeed, at a slight stretch, be described in such ways. As Wittgenstein conceives them, objects are precisely possible parts of facts. Objects are not items which have a reality independent of their possibilities for figuring in facts, so that we can ask: how do these things, being what they are, get to figure in facts in the ways they do? Again, an object's basic nature Wittgenstein calls its form, and the object's form is not something which grounds its possibilities for appearing in facts: it is those possibilities (TLP 2.0141).

Zalabardo appears however to be thinking in a more 'ontological' manner with his notion of 'basic', or 'fundamental'. So for example he asks whether "in treating facts as fundamental, Wittgenstein is ascribing this status to all states of affairs, obtaining and nonobtaining alike" (18). And the emphatic answer here is 'no'. A reading on which nonobtaining states of affairs are fundamental:

would be a serious distortion of Wittgenstein's view. It would amount, in effect, to ascribing to Wittgenstein an ontology of Russellian propositions, as they figure in the dual-relation theory .... Wittgenstein's thought that what is the case is fundamental ... is the claim that obtaining states of affairs are fundamental. (18)

Now there would seem to be a good question how, if objects are non-fundamental features shared between fundamental states of affairs, and if fundamentality belongs only to those states of affairs that obtain, objects could constitute the substance of the world which subsists independently of what is the case (TLP 2.021, 2.024). But the wider concern here is that I have little idea what Zalabardo might mean by 'fundamental', or indeed 'ontology'. Unlike the Russellian propositions of the early, dual-relation theory, Tractarian facts are not 'portions of reality' which either exist or fail to exist. So what is a claim that the fact that Jack loves Jill is fundamental to mean other than, perhaps, that this is an atomic fact (a state of affairs)? And what is a claim that the fact that Jack loves Jill is 'in Wittgenstein's ontology' to mean other than that this fact obtains - i.e. other than that Jack loves Jill?

5.

No doubt Zalabardo's thinking here connects to what I identified above as a realism of fact as prior to truth. And no doubt my bafflement connects to my understanding of Wittgenstein in which such a view isn't so much as on his radar. In any case, the divergence is I think far-reaching. The idea that Wittgenstein, like Russell, proposes a basic metaphysical framework given quite independently of thought, with his picture theory an account within that framework of a certain contingent (but for some reason very interesting) 'worldly phenomenon', namely representation, is I think liable to block access to the majority of the insights of his work.

As a final comment, I'd like to raise a question of the compatibility between the reactions of Zalabardo's Wittgenstein to the semantic and metaphysical problems. Zalabardo introduces the semantic problem as something which "arises for a strategy for explaining our faculty of propositional representation that exploits the resources of the 
CAF" (3). And he is explicit when discussing the metaphysical problem that Wittgenstein "rejects CAF" (14). So how is it that Wittgenstein presents a solution to the semantic problem (see, e.g., 11), as opposed to offering a view for which that problem doesn't arise? Zalabardo's own characterizations to one side, the tension here seems clear. If the semantic problem is to arise of what more is needed for representation besides the singling out of objects, then the act of singling out an object must have a status other than as an element of fact representation. But if singling out an object is something other than an element of fact representation, how nonetheless do objects have no status other than as elements (or 'features') of facts? I was unable to gather how Zalabardo intends this tension to be resolved.

References

Sullivan, P. (2005) 'Identity theories of truth and the Tractatus', Philosophical Investigations 28: 43-62

Colin Johnston, University of Stirling 